\title{
ALIGNING BIM AND GIS - CITYGML AND PLU TO ACHIEVE COMPLIANCE CHECKING MODEL
}

Elio HBEICH

Information System and Applications Division, CSTB, Sophia Antipolis 06560, France

Elio.hbeich@cstb.fr

Ana-Maria ROXIN

Université de Bourgogne Franche-Comté (UBFC) - LIB EA7534

ana-maria.roxin@ubfc.fr

Nicolas BUS

Information System and Applications Division, CSTB, Sophia Antipolis 06560, France nicolas.bus@cstb.fr

\begin{abstract}
Compliance checking for building models, cities and territories involves formalizing a set of model schemas and constraints. In this context, we aim at, on the one hand, conceiving a multi-scale model combining BIM and GIS information using semantic technologies (federated approach) and, on the other hand, at transforming urban regulations rules into SPARQL queries to allow querying and checking the level of compliance of the considered building. In this article we are introducing urban rules, define how they are connected to GIS and BIM. We give first present previous work related to transforming natural language rules into SPARQL queries, then we define directions to be addressed by our future work.
\end{abstract}

Keywords: urban rules, SPARQL, compliance checking

JEL classification: R14, R28, R52

DOI: $10.12948 / \mathrm{ie} 2019.04 .04$

\section{Introduction}

In France, urban construction, renovation or landscaping projects always require to check the considered municipality PLU (Local Urban Plan). This document indicates the urban planning rules governing the considered municipality, whether in terms of urban development, preservation of spaces and architectural prescriptions. The objective of the PLU is to improve: a) the living spaces of inhabitants with respect to a sustainable idea of urbanism; b) travel and transportation by taking into account infrastructure projects, c) neighborhoods restructuring while preserving natural heritage etc. The PLU is an operational and strategic document that defines the overall development plan of the municipality. It organizes the development of the municipality by fixing the urban planning rules, the different zones and the architectural prescriptions. It guaranties the balance between urban development and preservation of natural spaces in a perspective of sustainable development [1]. A PLU defines 4 main types of areas:

1. Urban areas (U): they are already equipped and urbanized;

2. Areas to be urbanized (AU): these are natural areas intended to accommodate future inhabitants or new businesses;

3. Agricultural zones (A): they are reserved for agricultural activities and housing for farmers only;

4. Natural and forest areas $(\mathrm{N})$ : they are preserved for their landscape.

Next sections of this article further detail these areas. 
www.conferenceie.ase.ro

\subsection{Urban Area}

Such zone corresponds to areas of the city that are urbanized or in the process of urbanization. Which means that the public networks (water, electricity, etc.) and the public road connect each parcel. That is why it is the most favorable area to build since it already has the necessary networks for viable land. In addition, when looking at the urban area of the PLU of medium or large cities, you will notice that zone $U$ is itself divided into sectors, notably zone UA, UB, UC, UD, UE, UL, UI, UP, UT, UV, and UY [2].

- Zone UA: mixed urban area of central character, predominantly residential, which may include shops, services, offices, craft activities, public facilities, compatible with a dense urban environment;

- Zone UB: is a mixed zone with a predominantly residential vocation, situated in extension of the village center, and composed mainly of individual housing isolated or constituted in the form of housing estates;

- Zone UC: Mixed urban area of central character, predominantly residential, which may include shops, services, offices, craft activities, public facilities, compatible with a dense urban environment;

- Zone UD: an urban area of low density and moderate urbanization, predominantly housing, which may include, for local needs, services, commercial, craft and public facilities compatible with existing housing;

- Zone UE: this is a mixed or medium-density urban area with a predominantly residential area, which may include shops, services, offices, handicrafts, public facilities, compatible with an urban environment;

- Zone UL: area of constructions and leisure facilities related to tourist activities;

- Zone UI: is an urban area whose main specificity is to host industrial and craft activities;

- Zone UP: is an area dedicated to port activity;

- Zone UT: it is an area for tourist and hotel accommodation as well as the shops and leisure facilities that accompany them;

- Zone UV: is an area intended for the reception of travelers;

- Zone UY: for craft, industrial, commercial and service activities.

\subsection{Areas to be urbanized (AU)}

The areas to be urbanized, known as "AU zones" are defined by Article R. 151-2029 of the French urban planning code. According to the French legislation, such area is considered as a zone intended to be open to public urbanization [3]. There are two types of AU zones:

- areas that can be immediately developed, mainly because of the presence of "existing sanitation on the immediate periphery of an AU area" and having "sufficient capacity to serve the buildings to be established throughout the area"; this zone is generally called "1AU";

- if this capacity is insufficient, the opening to urbanization is subject to a modification or revision of the PLU; this area is usually called "2AU".

\subsection{Agricultural areas (A)}

These are "areas of the municipality, equipped or not, to protect because of the potential agronomic, biological or economic agricultural land" (article R. 151-2230 of the French urban planning code) [4]. It is a strict and supervised regime, only the constructions or installations necessary for the public services and the agricultural exploitation are authorized there. In the agricultural zone of the PLU, you may authorize:

- Constructions and installations required for farming; 
www.conferenceie.ase.ro

- Housing essential for the farmer;

- Construction and installations required for public facilities or utilities (example: water tower).

Changing a building's destination from agricultural buildings into housing needs to be done according to local and precise regulation.

\subsection{Natural and forest areas (N)}

These are the "sectors of the commune, equipped or not, to be protected because of the quality of the sites, the natural environments, the landscapes and their interest, in particular from the aesthetic, historical or ecological point of view, or from the existence of a forest exploitation, or of their character as natural spaces" (article R. 151-2431 of the urban planning code) [5]. The natural areas of a PLU typically comprise the following elements:

- Constructions and installations necessary for the agricultural and forestry exploitation;

- Infrastructure dedicated to public services, as long as they do not affect the activity of the area. Demountable constructions and reception areas are also listed.

- If your house is located in a natural area of the PLU, your municipality may authorize the creation of an extension subject to full compliance with the landscape quality of the site.

The center of Figure 4 below shows the hierarchy and the architecture of PLU where each area, zone, subzone and district comes with several regulations that need to be respected when considering a construction process.

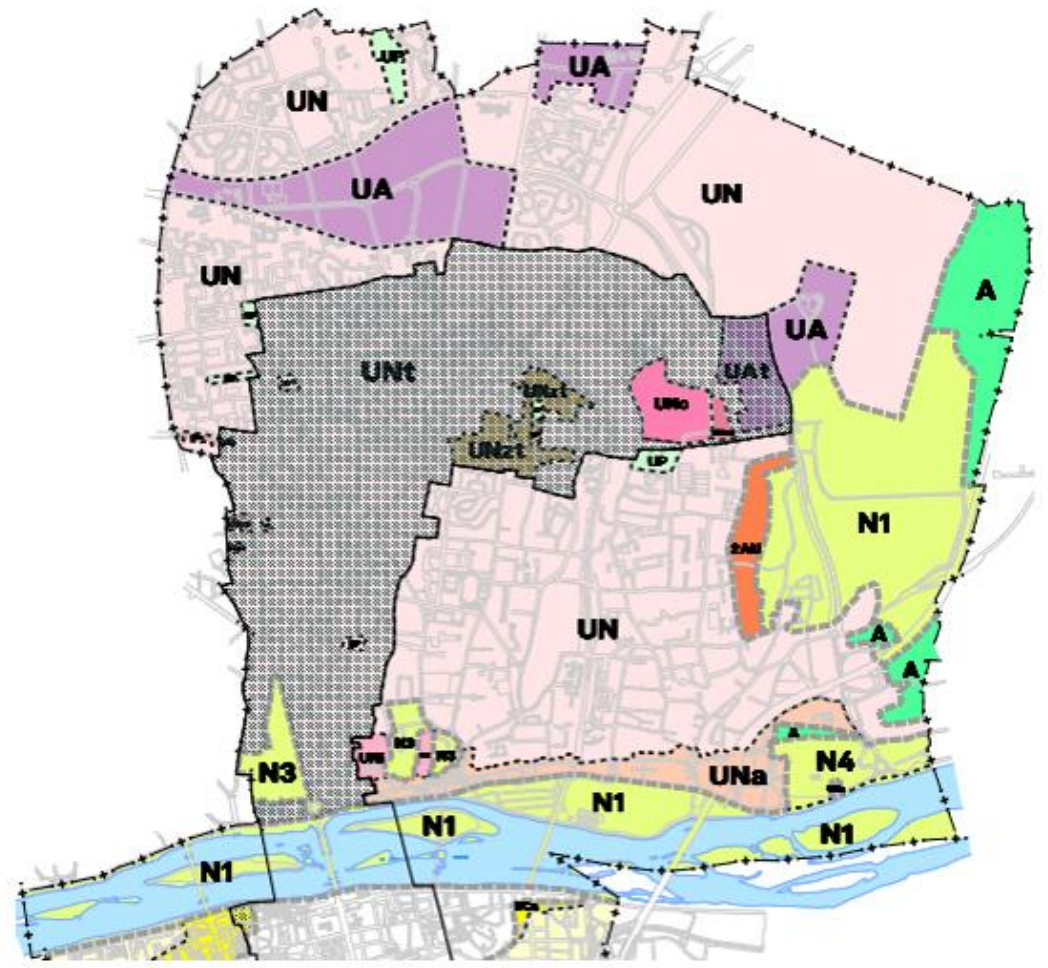

Figure 1. Illustration of multi-zone in a given area

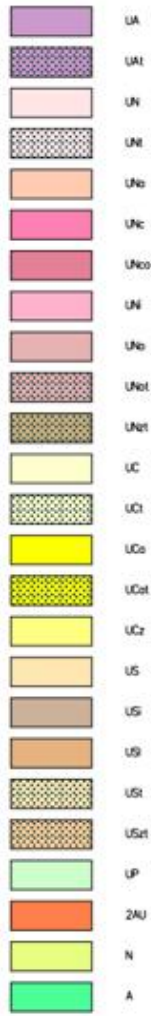


According to French law, the zoning plan is further interpreted in order to precisely define conditions to be respected by constructions and developments to be made in each zone of the municipality [6] [7]. Urban French regulations define the following elements:

- Building categories that are authorized by zone type;

- Requirements for developing municipality road, water and sanitation networks;

- The maximum footprint allowed for buildings;

- The setbacks and prospects to respect compared to public roads and neighborhoods;

- The maximum heights allowed for buildings;

- The conditions of treatment of façades and openings of buildings;

- Obligations for vehicle parking, as well as the number of parking locations to be created;

- Prescriptions for the managing and treating green spaces.

The table below shows an example of rules that need to be respected in each sub-zone:

Table 1. Example of rule defined in each zone [8]

\begin{tabular}{|c|c|c|c|c|c|c|c|c|c|}
\hline \multirow{2}{*}{\multicolumn{3}{|c|}{ PLU Marseille Provence }} & \multicolumn{3}{|c|}{ sUA Zone } & \multirow{2}{*}{$\begin{array}{c}\text { sUB } \\
\text { Zone } \\
\text { sUBf } \\
\end{array}$} & \multicolumn{3}{|c|}{ sUC Zone } \\
\hline & & & sUAd & sUAf & sUAq & & sUCr & sUCm1 & $\mathrm{sUCm} 2$ \\
\hline \multirow{2}{*}{$\begin{array}{l}\text { Quality } \\
\text { of free } \\
\text { spaces }\end{array}$} & \multirow{2}{*}{$\begin{array}{c}\text { Surface } \\
\text { of } \\
\text { green } \\
\text { spaces } \\
\text { and } \\
\text { open } \\
\text { spaces }\end{array}$} & $\begin{array}{l}\text { Total } \\
\text { green } \\
\text { space } \\
\text { area } \\
\end{array}$ & $\begin{array}{c}>= \\
30 \%\end{array}$ & $\begin{array}{c}>= \\
40 \%\end{array}$ & $>=15 \%$ & $\begin{array}{c}\text { No } \\
\text { regulation }\end{array}$ & $\begin{array}{c}>= \\
30 \%\end{array}$ & $\begin{array}{c}>= \\
40 \%\end{array}$ & $>=15 \%$ \\
\hline & & $\begin{array}{c}\text { Total } \\
\text { open } \\
\text { space } \\
\text { area } \\
\end{array}$ & \multicolumn{2}{|c|}{$\begin{array}{c}>=2 / 3 \text { total } \\
\text { area of green } \\
\text { spaces }\end{array}$} & $\begin{array}{l}\text { No } \\
\text { regulation }\end{array}$ & $\begin{array}{l}\text { No } \\
\text { regulation }\end{array}$ & \multicolumn{2}{|c|}{$\begin{array}{c}>=2 / 3 \text { total } \\
\text { area of green } \\
\text { spaces }\end{array}$} & $\begin{array}{l}\text { No } \\
\text { regulation }\end{array}$ \\
\hline
\end{tabular}

This indicates that we have a multi-area type, where each area is divided into multi-zones where different regulations are applied to check the compliance of city elements in the zone, e.g. buildings, transportation networks. This means that we have certain granularity and aggregation for the information contained in the PLU. To achieve an automated regulatory compliance checking for a construction project, we need to automatically extract requirements from various construction regulation documents, and transform these rules into a formalized syntax that enables machine automated reasoning. To reach our goal, we seek to express our set of rules in a language that can be handled and interpreted by a machine. As all considered urban models are converted into ontologies, we aim to transform natural language rule into SPARQL, as it is the equivalent of SQL for ontologies. In the next section we are presenting related work done in this specific context: transforming natural language rules into SPARQL.

\section{Related work}

In [10], authors present an approach and a tool to assist one in compiling regulations and technical documents. This approach addresses the construction industry and partially automates regulation conformance checking. For this purpose, a domain ontology, OntoDT, was introduced to represent concepts involved in the description of technical documents and regulations. Combining with SBVR (Semantics of Business Vocabulary and Business Rules) and Semantic Web languages, a controlled vocabulary was defined, based on which regulatory constraints extracted from the Practical Guides, edited by CSTB (French Scientific and Technical Centre for Building), were adapted. These two complementary representations are both based on the OntoDT ontology and are associated through RDF annotations. The SBVRbased approach reduces ambiguity, RDF and SPARQL based formalizations enable automating 
regulation conformance checking. Finally, the authors proposed a process model to organize regulations extracted from Technical Guides. However, the main limitation of this approach is that a large part of the regulations considered are implementation rules, which are easily represented in SBVR, but cannot be formalized in SPARQL.

In [10], authors present an information transformation (ITr) methodology. It relies on a rulebased, semantic natural language processing (NLP) approach. A set of semantic mapping $(\mathrm{SeM})$ rules and conflict resolution (CoR) rules are used to enable the automation of the transformation process. Several syntactic text features (captured using NLP techniques) and semantic text features (captured using an ontology) are used in the SeM and CoR rules. A bottom-up method is leveraged to handle complex sentence components. However, the approach has the following limitation: it was only tested on processing quantitative requirements, and does not address qualitative ones. The types of semantic patterns and conflicts in other types of requirements may lead to different performance results.

\section{Connecting PLU to Multi-scale model}

The figure below presents an example of urban plan of the French city Villepinte that is divided into multiple subzones. Each sub-zone has a certain regulation that applies: the subzones N, UEe, and UEf do not allow building construction, while the subzone UEb only allows building construction under certain conditions; the subzone UP allows any building construction. UP subzone implies that buildings respect several specific regulations such as the frontage height should not be above $55 \mathrm{~m}$, while the maximum building height is of $58 \mathrm{~m}$. In addition, the UP zone is divided to multiple districts, where each district has its own regulations specified as "alignment rules" e.g. the neighborhood should not create height and size contrast with the surrounding city, while the maximum floor number is of 5 . We now finally get to the building level where regulations specify that all the district should respect building construction regulations for example façade walls must be $18 \mathrm{~cm}$ wide and made of reinforced concrete.

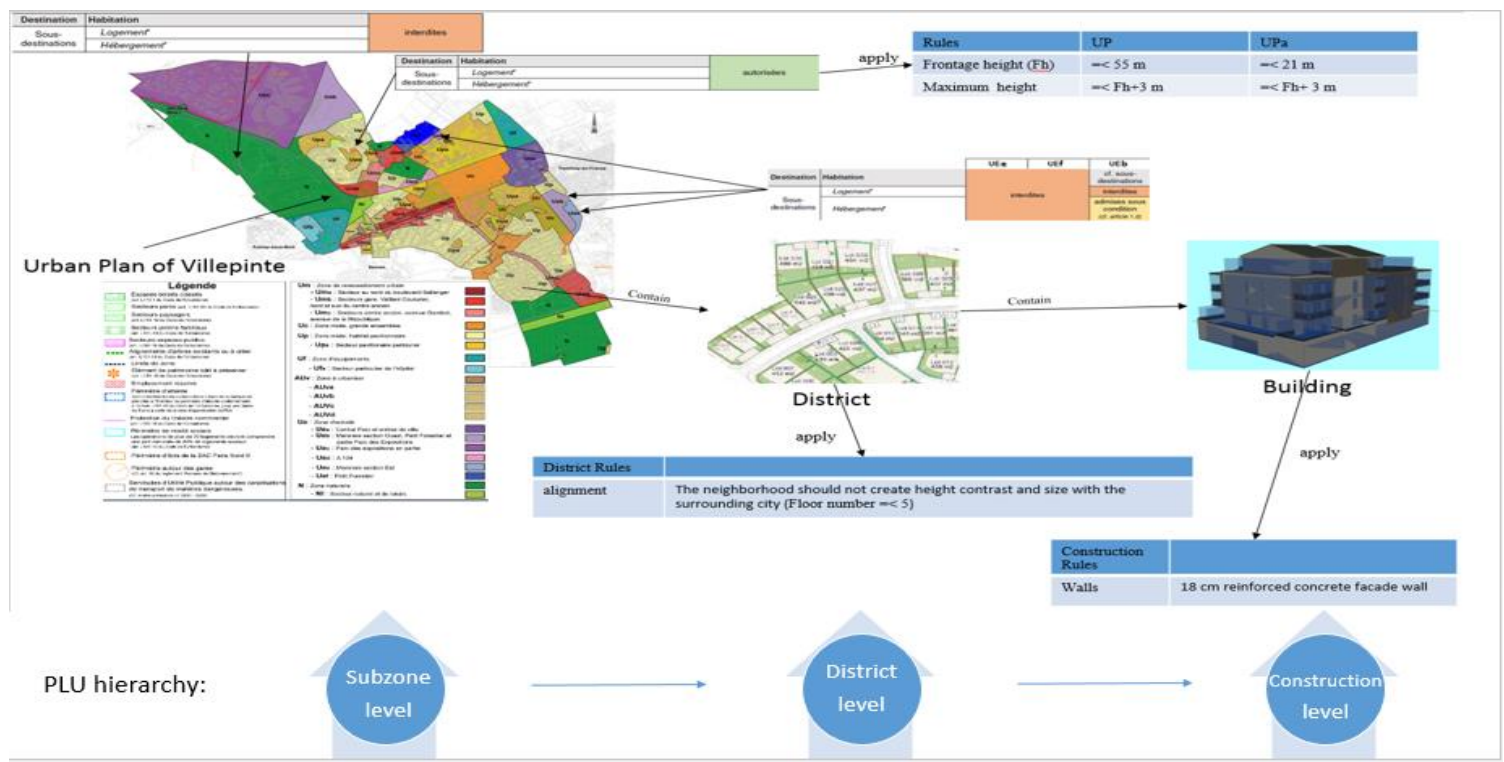

Figure 2. Hierarchy of rules

Because our model contains a certain information granularity, each level of detail in the multiscale model should be connected to a certain PLU level to generate the compliance checking report. On one hand BIM have one level of details and contain building information, on the other hand CityGML have 4 LODs and contain city information. We are aiming to establish an 
alignment between each model and PLU, such connection allows defining which information is contained in which Level of Detail (LOD) and specifies which rules are pertaining for each scale considered. For example, an IFC model and a CityGML LOD4 model are used to check building construction rules, while CityGML LOD2 and 3 along with district information are used to verify districts regulations per subzone.

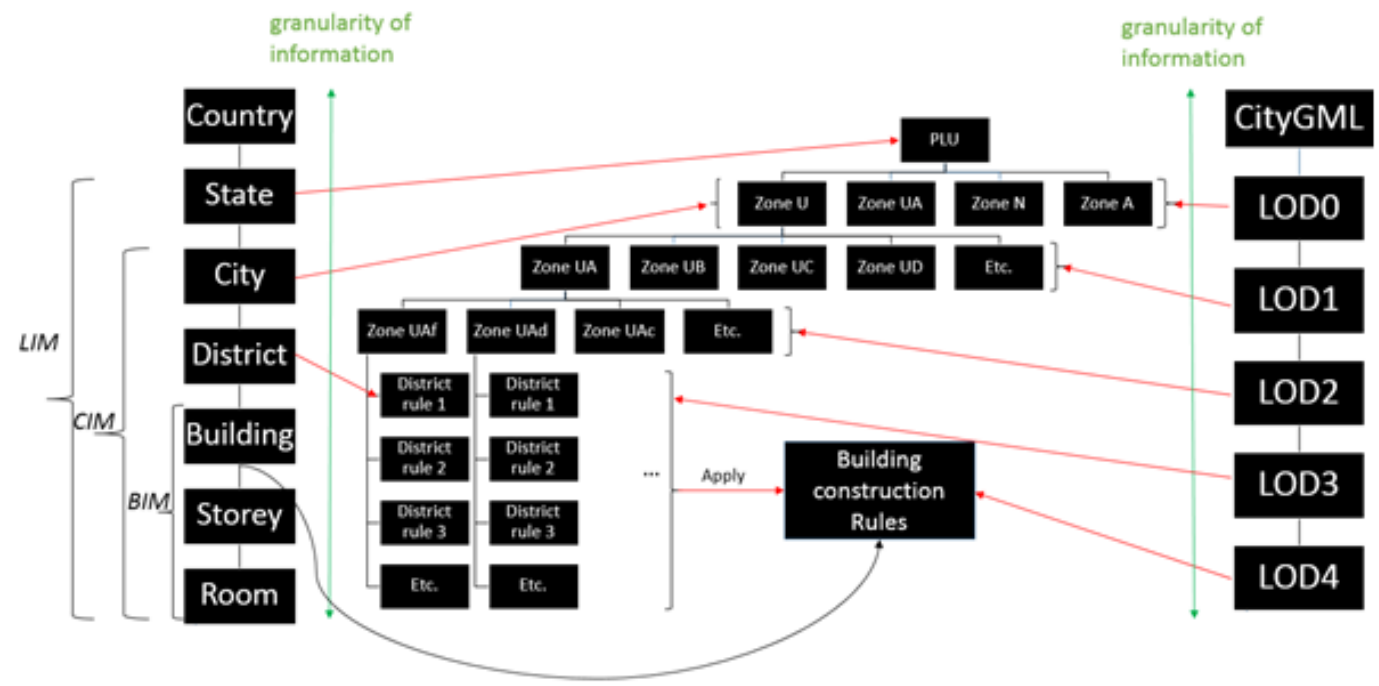

Figure 3. Connection BIM, SIG CityGML and PLU

\section{Conclusion}

This article has described how French urban regulations are structured into PLU hierarchies. When considering our overall goal which is conceiving a multi-scale semantic checker for urban environments, next steps will consider multi-scale models and conceive specific levels of details according to the different PLU level. Finally, based on these levels, we'll have to define alignment rules among them, while regulations will be implemented as granular logical rules that could be triggered according to the considered level or context. Our approach will be implemented and evaluated in the context of real-world PLUs and urban models.

\section{References}

[1] P. Newman, A. Thornley, "Urban Planning in Europe," 1996, London: Routledge, pp. 43.

[2] Code de l'urbanisme - Article R151-18. Code de l'urbanisme R151-18.

[3] Code de l'urbanisme - Article R151-20. Code de l'urbanisme R151-20.

[4] Code de l'urbanisme - Article R151-22. Code de l'urbanisme R151-22.

[5] Code de l'urbanisme - Article R151-24. Code de l'urbanisme R151-24.

[6] Code de l'urbanisme - Article R151-52. Code de l'urbanisme R151-52.

[7] Code de l'urbanisme - Article R151-53. Code de l'urbanisme R151-53.

[8] Plan local d'urbanisme intercommunal du territoire Marseille Provence, 2018.

[9] K. R. Bouzidi, B. Fies, C. Faron-Zucker, A. Zarli, N. L. Thanh, "Semantic Web Approach to Ease Regulation Compliance Checking in Construction Industry," Future Internet, no. 4, 2012, pp. 830-851.

[10] J. Zhang, N. M. El-Gohary, "Automated Information Transformation for Automated Regulatory Compliance Checking in Construction," Journal of Computing in Civil Engineering, vol. 29, B4015001, 2015. 